\title{
Analysis of genetic differentiation and genomic variation to reveal potential regions of importance during maize improvement
}

\author{
Xun $\mathrm{Wu}^{1,2}$, Yongxiang $\mathrm{Li}^{1}$, Xin $\mathrm{Li}^{1}$, Chunhui $\mathrm{Li}^{1}$, Yunsu Shi ${ }^{1}$, Yanchun Song ${ }^{1}$, Zuping Zheng ${ }^{2}, \mathrm{Yu} \mathrm{Li}^{1 *}$ \\ and Tianyu Wang ${ }^{1 *}$
}

\begin{abstract}
Background: Exploring genetic differentiation and genomic variation is important for both the utilization of heterosis and the dissection of the genetic bases of complex traits.

Methods: We integrated 1857 diverse maize accessions from America, Africa, Europe and Asia to investigatetheir genetic differentiation, genomic variation using 43,252 high-quality single-nucleotide polymorphisms(SNPs),combing GWAS and linkage analysis strategy to exploring the function of relevant genetic segments.

Results: We uncovered many more subpopulations that recently or historically formed during the breeding process. These patterns are represented by the following lines: Mo17, GB, E28, Ye8112, HZS, Shen137, PHG39, B73, 207, A634, Oh43, Reid Yellow Dent, and the Tropical/subtropical (TS) germplasm. A total of 85 highly differentiated regions with a $D_{\text {EST }}$ of more than 0.2 were identified between the TS and temperate subpopulations. These regions comprised $79 \%$ of the genetic variation, and most were significantly associated with adaptive traits. For example, the region containing the SNP tag PZE.108075114 was highly differentiated, and this region was significantly associated with flowering time (FT)-related traits, as supported by a genome-wide association study (GWAS) within the interval of FTrelated quantitative trait loci (QTL). This region was also closely linked to zcn8 and vgt1, which were shown to be involved in maize adaptation. Most importantly, 197 highly differentiated regions between different subpopulation pairs were located within an FT- or plant architecture-related QTL.

Conclusions: Here we reported that 700-1000 SNPs were necessary needed to robustly estimate the genetic differentiation of a naturally diverse panel. In addition, 13 subpopulations were observed in maize germplasm, 85 genetic regions with higher differentiation between TS and temperate maize germplasm, 197 highly differentiated regions between different subpopulation pairs, which contained some FT- related QTNs/QTLS/genes supported by GWAS and linkage analysis, and these regions were expected to play important roles in maize adaptation.
\end{abstract}

Keywords: Genomic variation, Subpopulation differentiation, Zea mays $L$

\section{Background}

Maize (Zea mays L.) is widely planted throughout the world, including in more than 70 countries across six continents [1]. Maize originated in south-central Mexico [2] and spread throughout the Americas for thousands of years before it was introduced to Europe, Africa, and Asia after Columbus discovered the New World [3]. During

\footnotetext{
*Correspondence: liyu03@caas.cn; wangtianyu@caas.cn

${ }^{1}$ Institute of Crop Science, Chinese Academy of Agricultural Science, Beijing, China

Full list of author information is available at the end of the article
}

this spread, maize continually improved via natural and artificial selection in order to adapt to different environments [4]; a number of landraces and inbreds were developed [5], and many hybrids with high yields have been released to satisfy the increasing need of humans [6].

In the past several decades, maize's diffusion [3], improvement [7-11], pedigrees [12, 13], and genetic basis for phenotypic variations [14-16] have been well documented, providing scientific proof for the genetic contributions to historical yield increases and the formation of heterotic groups. For instance, American maize 
germplasms were divided into three main heterotic groups: Iowa Stiff Stalk Synthetic (SS), Non-Stiff Stalk (NSS), and Iodent (IDT) [10]. African maize germplasms were divided into three clusters: Meso-American landraces, Coastal Brazilian landraces, and Tropical varieties [17]. Chinese maize germplasms are divided into five subpopulations: including Ludahonggu, TSPT, P group, Reid and Lancaster [18-20]. The aforementioned studies provided useful information for both heterosis utilization and the dissection of the genetic basis for complex traits, but the accessions used in previous studies were obtained from a single geographical origin and have relied on the smallest number of markers, which limits our understanding of genetic differentiation. The development of high-throughput genotyping strategies has facilitated the study of historical genetic changes in maize [21-23]. Recently, another large natural panel of 2,815 maize accessions was investigated using the genotyping by sequencing (GBS) method [12], and this study provided abundant information about pair relationships of accessions and identified many new genetic loci associated with flowering time (FT)-related traits. Five subpopulations were observed in this paper; the distance between SS and NSS subpopulations was small, which indicated a slight bias when comparing with previous studies and the knowledge of maize pedigrees based on breeding practice [10, 22, 24-26].

In addition, many studies of genomic variation reported using $G_{S T}$ and its relatives $\left(D_{\mathrm{EST}}, \mathrm{F}_{\mathrm{ST}}\right.$ ) [27]. Haag et al. [28] demonstrated that $\mathrm{D}_{\mathrm{EST}}$ constituted an alternative measure of genetic differentiation between populations. However, the traditional $\mathrm{F}_{\mathrm{ST}}$ value has been widely used to estimate plant genetic differentiation. A higher $\mathrm{F}_{\mathrm{ST}}$ of selected features was observed between subpopulations using 284 maize inbreds from Minnesota [22], and this value was larger than that between temperate maize germplasms [9]. Romay et al. [12] showed that most of germplasms from classic breeding programs of the Corn Belt were closely related, with an average pair-wise $\mathrm{F}_{\mathrm{ST}}$ of 0.04 , which was larger than the 0.027 value reported between tropical and temperate lines [29] and the 0.02 value reported between landraces and improved lines. Nevertheless, this value did not exceed the 0.11 value reported between teosinte and landraces [30] However, most studies have previously only reported the differentiation phenomenon and extent of genetic variation between subpopulations. The potential genomic regions of importance that are highly differentiated and associated with putative function are poorly understood, especially for maize.

In this paper, we integrate maize germplasms from America, Africa, Europe and Asia, including 1857 accessions from more than sixteen countries worldwide, and present an in-depth analysis of genetic differentiation and genomic variation using a dataset of 43,252 singlenucleotide polymorphisms (SNPs). We dissected the genome-wide variation patterns of selection fixation, uncovered the subdivision of population structure, identified highly differentiated genomic regions between subpopulations, combined genome-wide association studies (GWAS) of FT-related traits, and compared the results with public data on the quantitative trait loci (QTL) and bioinformatics analysis to identify adaptive genomic regions and relevant candidate genes that may have been important during maize development and the formation of modern heterotic groups.

\section{Results}

\section{Ascertainment bias}

The average correlation coefficients of the first five principal components (PCs) between one given subset and the entire set with all markers are shown in Additional file 1: Figure S1. The correlation coefficients between the subset and the entire set sharply increased from 0.65 for a marker number of 500 to 0.97 for a marker number of 700 . A second sharp increase emerged when the marker number increased from 800 to 1000, with a corresponding increase in the correlation coefficient from 0.97 to 0.99 . Furthermore, the correlation coefficient did not significantly change when the marker number increased from 2000 to 43,252 . The results indicated that 1,000 SNPs might be sufficient for population structure analyses.

\section{Model-based population structure}

The subpopulations of 1857 accessions based on the admixture model-based algorithm were analyzed in depth using the even distribution of 5000 SNPs. The results are depicted in Fig. 1. The delta $K(\Delta K)$ peak was maximized when $\mathrm{k}=2$ (Fig. 1a), indicating that the accessions could be categorized into two groups: tropical/subtropical (TS) germplasm and temperate germplasm (Fig. 1b $\mathrm{k}=2$ ). A second peak of $\Delta \mathrm{K}$ emerged at $\mathrm{k}=4$ (Fig. 1a), indicating that this panel could be further divided into four subgroups: SS, NSS, Modified Introduction in China (MICN), and TS I (Fig. 1b k=4). Notably, MICN formed during the long history of maize breeding in China because Chinese maize breeders have developed a number of inbred lines derived from Chinese landraces and U.S. hybrids. These varieties significantly differ from U.S. inbreds [19]. A third peak of $\Delta \mathrm{K}$ was observed at $\mathrm{k}=7$ (Fig. 1a), indicating that this panel could be comprehensively categorized into seven subpopulations, each including one of the following representative lines: B73, Huangzaosi (HZS), 207, Oh43, Mo17, Shen137, and some from TS regions (Fig. 1b, $k=7$ ). Detailed information for each accession is listed in Additional file 2: Table S1. 


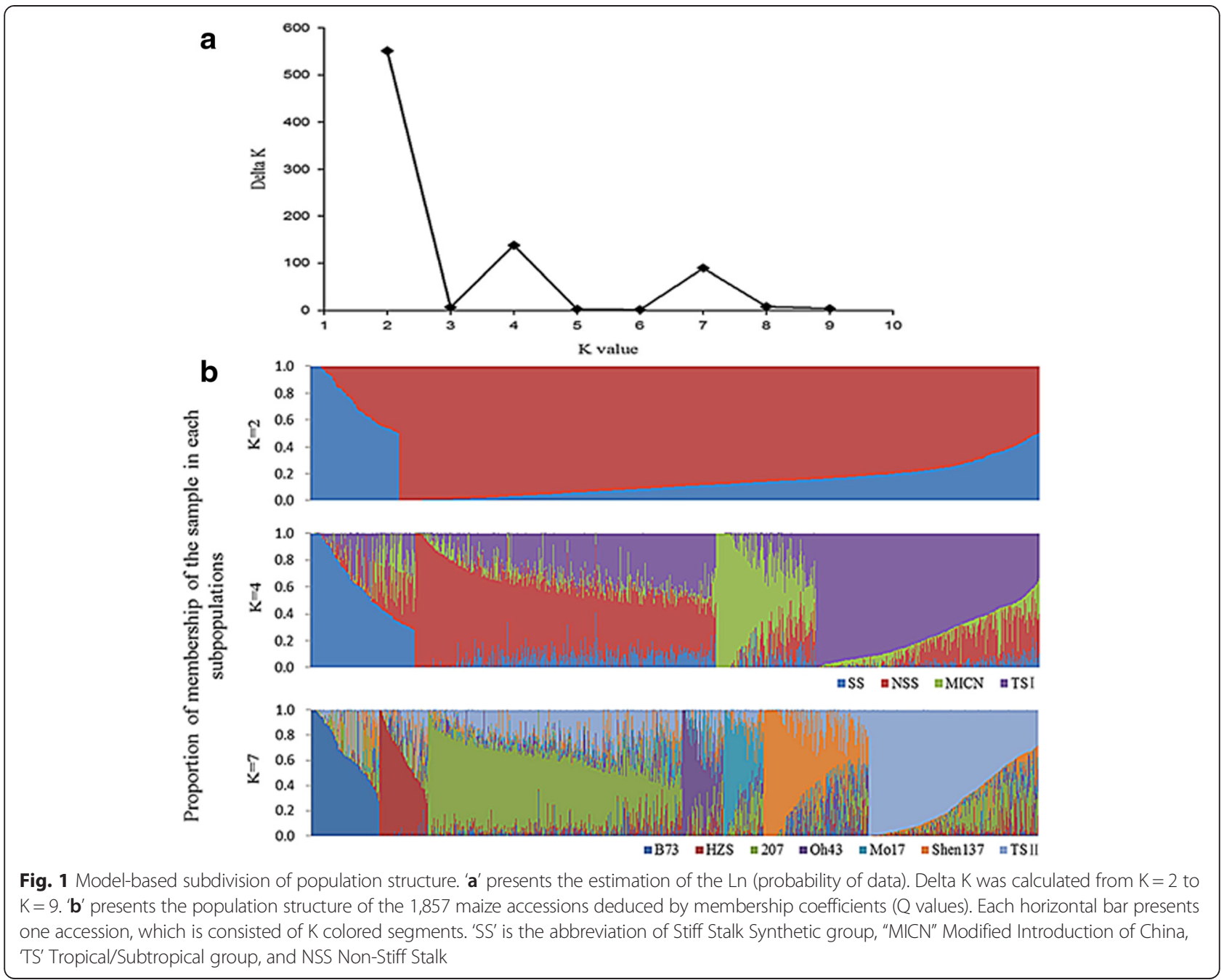

\section{Clustering analysis}

A neighbor-joining tree was constructed based on the modified Euclidean distance and is shown in Fig. 2. The 1857 accessions were clustered into two major groups according to their origins: the TS and Tem-tropic subpopulation. The TS subpopulation contained 525 accessions, including 195 accessions from Mexico, 187 from the U.S., 77 from China, 17 from Sudan, 10 from Thailand, 9 from Canada, 9 from Tanzania, 6 from Nigeria, 3 from Somalia, 3 from Benin, 3 from Zambia, 3 from Chad, 2 from Spain, 1 from Ghana, 1 from Germany, 1 from Yugoslavia, and 1 from Egypt (Additional file 2: Table S1). The Tem-tropic subpopulation contained 1,332 accessions, which could be further clustered into four subpopulations, SS, NSS, Iodent (IDT) and TS, according to their origins and pedigrees. A further analysis showed that the accessions from these four subpopulations could be clustered into 13 subgroups, with the following representative lines: Reid Yellow Dent, Oh43, A634, 207, B37, B73, PHG39, Shen137, Huangzaosi (HZS), Ye8112, E28, GB and Mo17 (Fig. 2).

\section{Principal component analysis (PCA)}

The PCA results showed comprehensive patterns of subpopulation and a good agreement with both model-based population structure and clustering analyses (Fig. 3). The entire panel of 1857 accessions exhibited moderate differentiation and some overlap between the temperate and TS germplasm; representative lines from the TS and temperate region significantly differed, e.g., B73 from the temperate and Ki3 from the TS region of Thailand, but the accessions from the adjacent regions did not markedly differ. Which may be resulted by the lager introgression existing between temperate and tropical/subtropical accessions and lower power of PCA in population structure analysis by using only two PCs. The accessions from the temperate subpopulation were further categorized into the B73 subpopulation according to the results of model-based structure analysis (Fig. 3b) or the Ye8112, B37 and A634 subpopulations based on the results of modified Euclidean distance (Fig. 3c). 


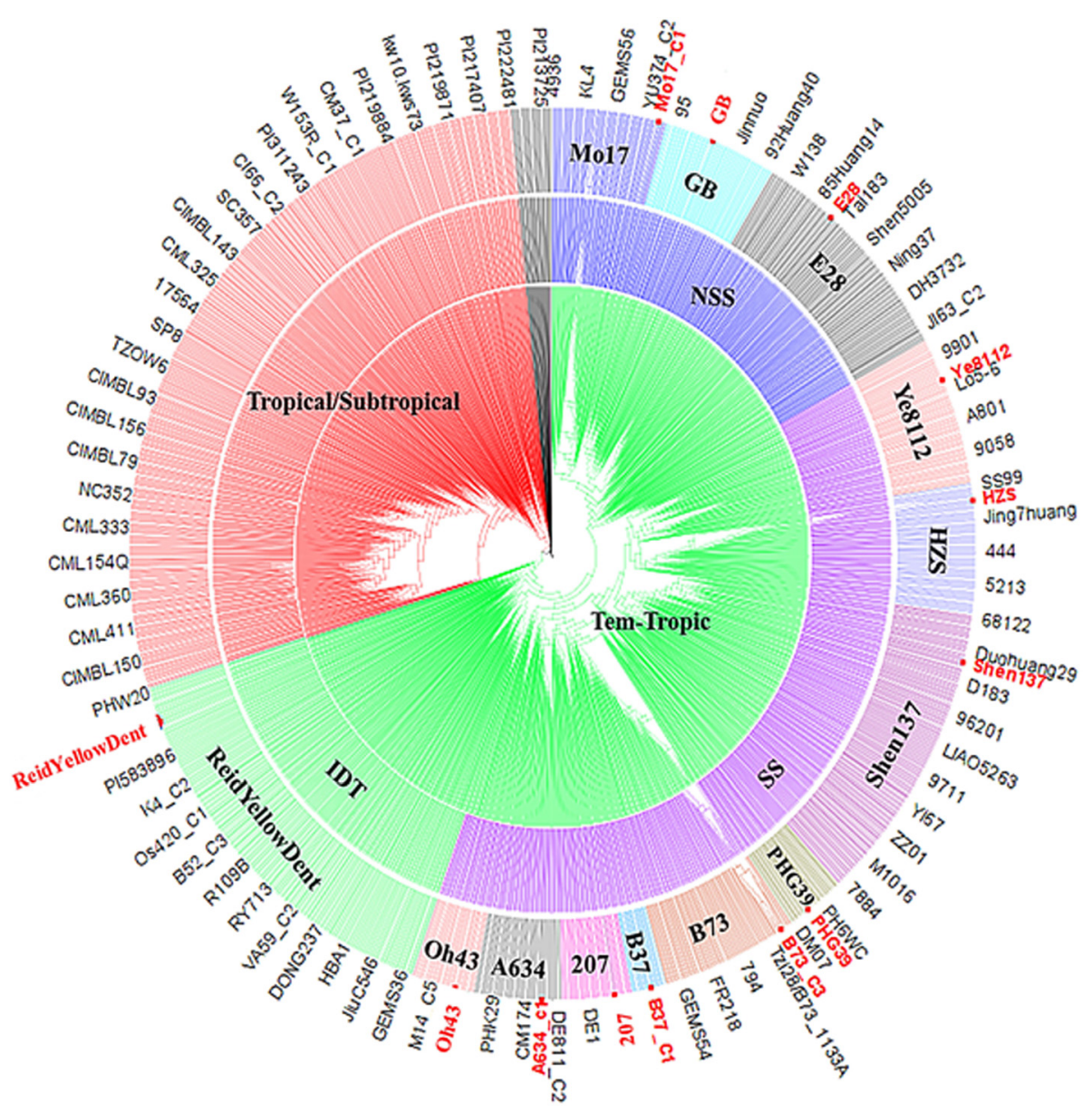

Fig. 2 Neighbor-joining trees of the 1,857 maize accessions. Mo17 is a representative line of Non-Stiff Stalk (NSS). GB is a representative line derived Chinese landrace. E28 is a representative line of the Ludahonggu group. Ye8112 a representative line of the Modified Reid group. 'HZS' is an abbreviation of Huangzaosi, which is a representative line of the Tangsipingtou group (TSPT). Shen137 is a representative line of the PA group. PHG39 is a parent derived from Argentine Maize Amargo background. B73 is a representative line of Stiff Stalk Synthetic (SS). B37, 207, A634, Oh43, and Reid Yellow Dent are the representative lines of different subpopulations, respectively

Based on the pedigrees, most lines were from the U.S. and China (Fig. 3d and Additional file 2: Table S1). In addition, the TS population was further divided into the HZS, 207, Oh43, Mo17 and Shen137 subpopulations based on the model-based population structure, which corresponded to HZS, GB, Shen137, Mo17, and Reid Yellow Dent based on a clustering analysis (Fig. 3c). These subpopulations contained inbred lines of a TS lineage in their pedigrees or lines from CIMMYT, Mexico and other tropical regions (Fig. 3a and d). Moreover, many accessions were categorized into new groups, such as the PHG39, 207, A634, Oh43, B37 and E28 subpopulations; most accessions in these groups originated from regions between temperate and TS zones (Fig. 3) due to the introgression of TS genotypes into regions of temperate germplasms.

\section{Summary statistics of genetic diversity}

The accessions of the entire panel of 1857 accessions were moderately similar, with more than $96.22 \%$ of the pair-kinship coefficients varying from 0.30 to 0.53 (Fig. 4a). The average linkage disequilibrium (LD) distance was 30 kilo-bases $(\mathrm{kb})$, varying from 20 to $50 \mathrm{~kb}$, with an $\mathrm{r}^{2}$ exceeding 0.1 (Fig. $4 \mathrm{~b}$ ). Combining the results of both the model-based population structure and genomic variation analyses indicated pronounced patterns of genetic variation among different subpopulations. These patterns were fixed by artificial or natural selection and resulted in the division of subpopulations during breeding. The TS subpopulation was more genetically diverse than the temperate subpopulation, with gene diversities (GDs) of 0.364 and 0.284 , respectively, and polymorphism information contents (PICs) of 0.281 and 0.231 , respectively (Table 1 ). Similar trends 


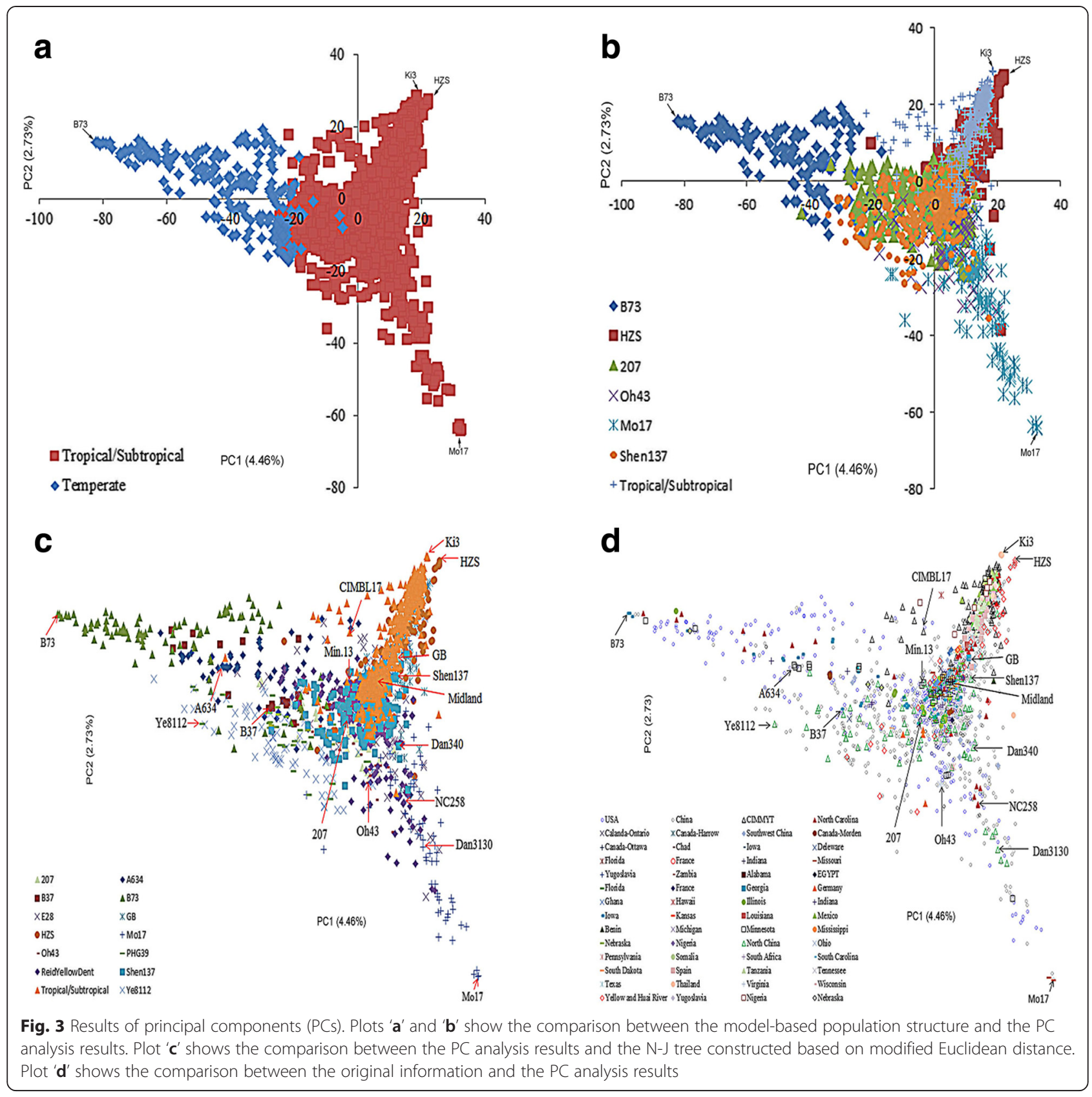

were validated with a smaller proportion of SNPs in LD for TS when comparing with a larger proportion of SNPs in LD for the temperate subpopulation (Fig. 4c).

\section{Genomic differentiation between subpopulations}

The proportion of genetic variance due to subpopulations $\left(D_{\mathrm{EST}}\right)$ was measured to interpret the genomic variation between subpopulations (Table 2, Fig. 4(d), Fig. 5 and Additional file 1: Figure S2). The $\mathrm{D}_{\mathrm{EST}}$ indicated different patterns of genomic differentiation between the subpopulations, ranging from 0 to 0.39 between TS and Temperate (average 0.08), from 0 to
0.45 between TS I and SS (average 0.09), from 0 to 0.45 between SS and NSS (average 0.07), from 0 to 0.41 between NSS and MICN (average 0.05), from 0 to 0.38 between MICN and TS I (average 0.06), from 0 to 0.30 between NSS and TS I (average 0.03), and from 0 to 0.57 between SS and MICN (average 0.08). The SS and TS I varieties were more differentiated, with 332 genomic regions having a large $\mathrm{D}_{\mathrm{EST}}$ (twice the average level) (Fig. 5a). Furthermore, 250 genomic regions were highly differentiated between SS and MICN, 235 were highly differentiated between TS and Temperate, 92 were highly differentiated between MICN and TS I, 51 

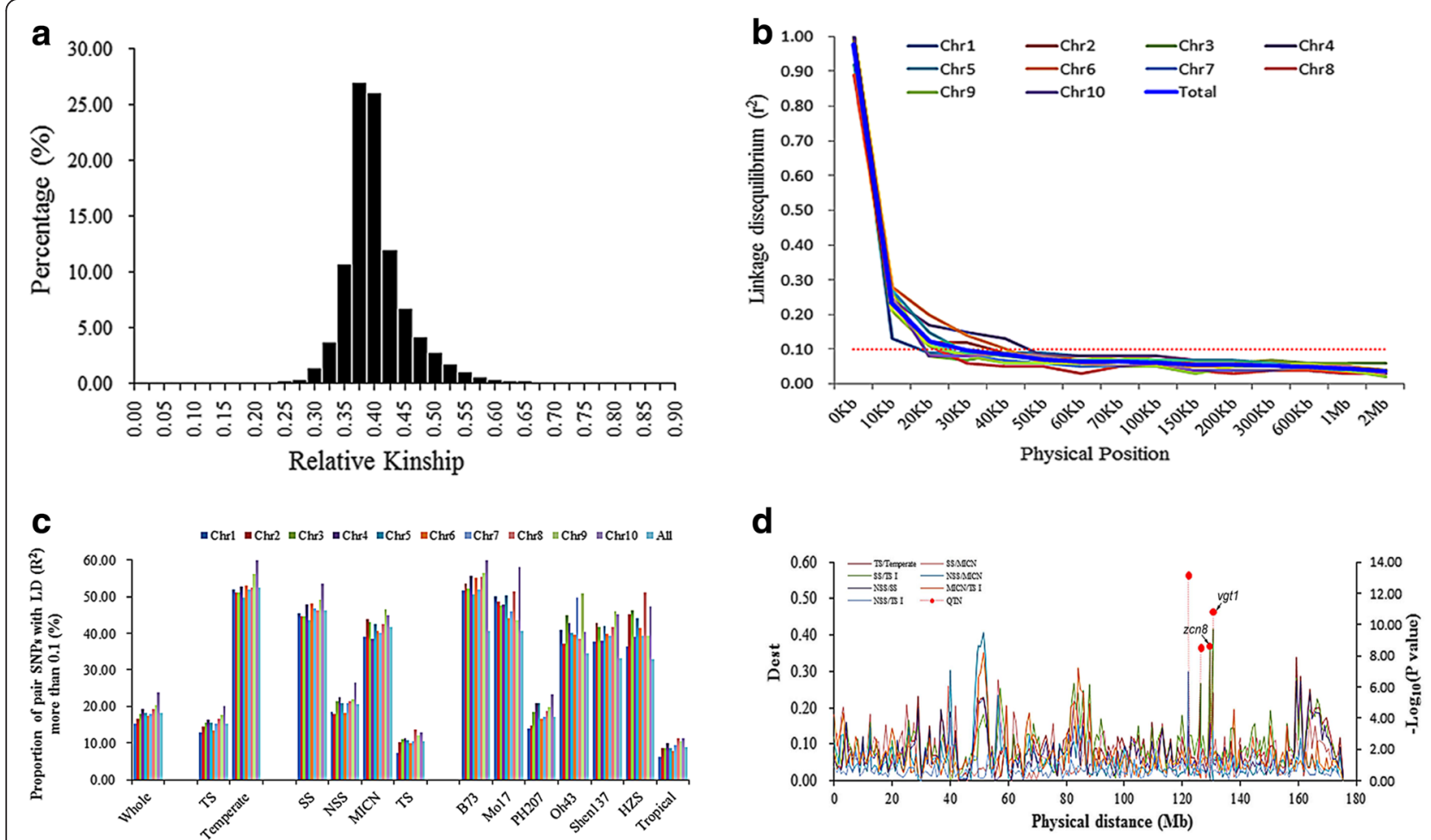

Fig. 4 Summary statistics of genetic variation existing in the whole set of accessions. 'a' is a picture of pair-wise kinship of the 1857 accessions. 'b' displays the decay level of linkage disequilibrium (LD) on different chromosomes and across the whole genome. 'c' shows the comparison of LD level between different subpopulations. ' $\mathbf{d}$ ' pictures the genomic differentiation on Chromosome 8

were highly differentiated between NSS and MICN, and 8 were highly differentiated between NSS and TS I, with a $D_{E S T}$ of more than twice the average level. Most importantly, 85 highly differentiated regions with a $\mathrm{D}_{\mathrm{EST}}$ exceeding 0.2 were identified between the TS and the temperate subpopulations. Of these 85 regions, 68 were located within the interval of plant architecture or FTrelated QTL, and two regions were closely linked to vgt1 and $z$ cn 8 (Additional file 2: Table S2 and S3). Furthermore, a number of special genomic regions were also found to be highly differentiated. In particular, subpopulation pairs and common regions were identified among different population pairs (Fig. 5b). In total, 303 genomic regions with a high $D_{E S T}$ of more than 0.2 were detected, and these regions were located within 197 FT- or plant architecture-related QTL. For example, the region containing the tag SNP PZE.108075114 differed more between the TS and temperate subpopulations and was associated with a $D_{\text {EST }}$ of 0.32 ; this region was located within an FT-related QTL cluster and contained the flanking markers PHTi060 and bnlg1599 (Additional file 2: Table S3).

\section{Genome-wide study of FT-related traits}

The phenotypes of FT-related traits were significantly positively correlated between the environments (Additional file 1: Figure S3). Thus, the BLUPs for each accession across the three environments were calculated, and the phenotype-genotype associations were analyzed. To validate the putatively adaptive function of highly differentiated target regions, we used the FT-related traits DTT, DTS, and DTP to perform a GWAS with 43,252 SNPs as a case study. The results indicated that some highly differentiated genomic regions were associated with FT-related traits. For

Table 1 Summary statistics of genetic diversity

\begin{tabular}{|c|c|c|c|c|c|c|c|c|c|c|c|c|c|c|}
\hline \multirow[t]{2}{*}{ Index } & \multirow[t]{2}{*}{ Total } & \multicolumn{2}{|l|}{$K=2$} & \multicolumn{4}{|l|}{$K=4$} & \multicolumn{7}{|l|}{$K=7$} \\
\hline & & TS & Temperate & SS & NSS & MICN & TS I & B73 & Mo17 & $\mathrm{PH} 207$ & Oh43 & Shen137 & $\mathrm{HZS}$ & TS ॥ \\
\hline Gene Diversity & 0.365 & 0.364 & 0.284 & 0.301 & 0.361 & 0.306 & 0.348 & 0.268 & 0.299 & 0.360 & 0.294 & 0.311 & 0.272 & 0.345 \\
\hline $\mathrm{PIC}$ & 0.291 & 0.291 & 0.231 & 0.244 & 0.289 & 0.247 & 0.279 & 0.219 & 0.242 & 0.287 & 0.239 & 0.250 & 0.223 & 0.277 \\
\hline Heterozygosity & 0.046 & 0.048 & 0.025 & 0.027 & 0.058 & 0.047 & 0.037 & 0.023 & 0.033 & 0.065 & 0.028 & 0.049 & 0.034 & 0.033 \\
\hline
\end{tabular}

Note: $\mathrm{K}$ is the number of subpopulations. 'TS' is an abbreviation of Tropical/Subtropical subpopulation. 'SS' is an abbreviation of Stiff Stalk Synthetic subpopulation. 'NSS' is an abbreviation of Non-Stiff Stalk. 'MICN' is an abbreviation of Modified Introduction of China 
Table 2 Variation of $D_{E S T}$ between subpopulations

\begin{tabular}{|c|c|c|c|c|c|c|c|}
\hline $\mathrm{K}=2$ & Temperate & TS & & & & & \\
\hline Temperate & 0.000 & 0.170 & & & & & \\
\hline TS & & 0.000 & & & & & \\
\hline$K=4$ & SS & NSS & $\mathrm{MICN}$ & TS I & & & \\
\hline SS & 0.000 & 0.164 & 0.146 & 0.172 & & & \\
\hline NSS & & 0.000 & 0.134 & 0.053 & & & \\
\hline MICN & & & 0.000 & 0.121 & & & \\
\hline TS & & & & 0.000 & & & \\
\hline$K=7$ & 207 & B73 & $\mathrm{HZS}$ & Mo17 & Oh43 & Shen137 & TS ॥ \\
\hline 207 & 0.000 & 0.251 & 0.283 & 0.260 & 0.242 & 0.113 & 0.059 \\
\hline B73 & & 0.000 & 0.295 & 0.309 & 0.246 & 0.202 & 0.247 \\
\hline $\mathrm{HZS}$ & & & 0.000 & 0.242 & 0.194 & 0.199 & 0.208 \\
\hline Mo17 & & & & 0.000 & 0.180 & 0.182 & 0.226 \\
\hline Oh43 & & & & & 0.000 & 0.156 & 0.194 \\
\hline Shen137 & & & & & & 0.000 & 0.110 \\
\hline TS ॥ & & & & & & & 0.000 \\
\hline
\end{tabular}

example, the SNP of PZE-108070380 was significantly associated with DTT $\left(P=7.05 \times 10^{-14}\right)$, DTP $\left(P=2.57 \times 10^{-9}\right)$ and DTS $\left(P=2.12 \times 10^{-8}\right)$ (Fig. 6). This SNP was located within the $z c n 8$ gene, which is involved in maize migration from tropical to temperate regions [31]. The SNP PZE-108076585 was significantly associated with DTS $\left(P=1.43 \times 10^{-11}\right) \quad$ (Fig. 6). This SNP was located within the vgt1 gene, which is involved in maize adaptation [32]. Furthermore, twelve other SNPs were also strongly associated with FT-related traits (Fig. 6), and the regions surrounding these SNPs were more differentiated than the rest of the genome (Fig. 4d, Additional file 1: Figure S2, Additional file 2: Table S2).

\section{Discussion}

\section{Moderate SNPs are reliable in interpreting population structure division}

Previous reports compared the effect of different marker systems and concluded that the subdivision of populations depended on the marker size and population [18, 33-35]. For instance, when 884 SNPs were used in one association panel of 154 inbred lines, more than $26.4 \%$ of lines were allocated to the mixed group. This rate was higher than the $20.6 \%$ rate identified by using 84 simple sequence repeat (SSR) markers [35]. Comparing the effect between 847 SNPs and 89 SSRs in one panel of 254 inbred lines yielded similar results [36], they proposed that many more SNPs would be required to study population structure. Here, we compared the average correlation coefficients of division for subpopulations between one given subset with different marker sizes and the entire set with all markers; we used SNPs varying from 500 to 43,252 in a panel of identical samples. The results showed that 700 SNPs are sufficient to reliably divide subpopulations in this panel, with an average correlation coefficient of the first five PCs of 0.97 between the subsets and the entire set of SNPs. The average correlation coefficient could be increased to 0.99 by increasing the number of SNPs to 1000 (Additional file 1: Figure S1). $\mathrm{Yu}$ et al. [37] reported moderate genetic diversity with a PIC of 0.24 for a sample size of 274 . We herein report a
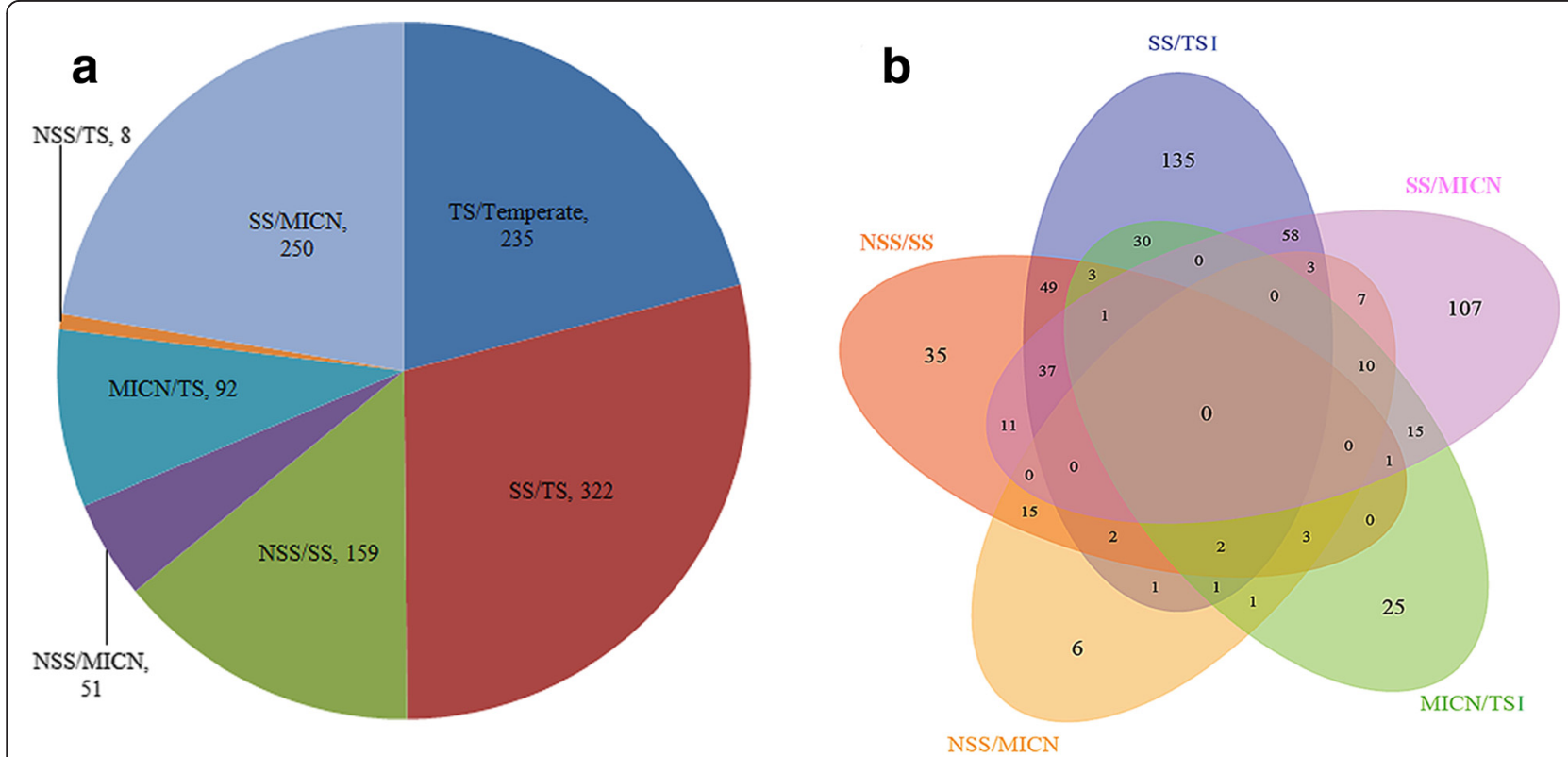

Fig. 5 Counts of genetic regions with high differentiation. 'a' shows the counts of genomic regions for each subpopulation pair. 'b' shows the comparison of genomic regions with high differentiation among different subpopulation pairs 


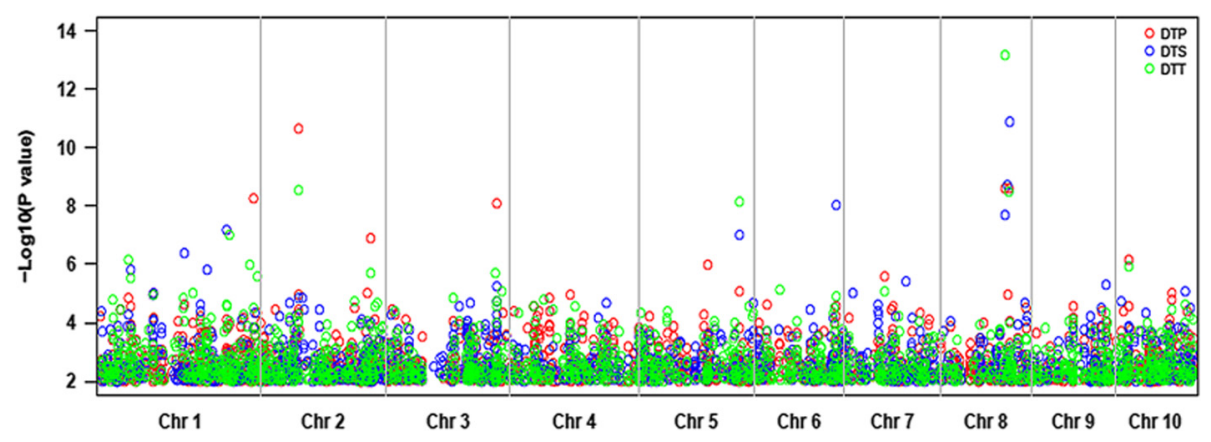

Fig. 6 Manhattan plot of GWAS results for flower time related traits. Red cycle refers to days to pollen-shedding (DTP), blue cycle shows days to silking (DTS), and green cycle shows days to tasseling (DTT). Red line shows the cutoff value of 5.94 (defined as: $-\log _{10}(0.05 / 43,252)$ )

similar PIC of 0.29 for a sample size of 1,857 . Yu et al. [37] demonstrated that more than 1000 SNPs are necessary needed to robustly estimate the genetic differentiation of a naturally diverse panel, and this requirement exceeded the 700-1000 SNPs found to be necessary herein. Thus, a larger sample size is expected to significantly improve the detection power of subdivisions in the populations. These results were consistent with those reported by Morin et al. [33], who compared the subpopulation differentiation for sample sizes ranging from 10 to 100. The results reported herein suggested that a moderate SNP marker number $(700-1000)$ is sufficient to divide population structures in this panel.

\section{Comprehensive patterns of population structure in maize inbreds worldwide}

The analysis of population structure is an important step in dissecting the genetic basis of complex traits via association analyses [38]. Such an analysis can result in false positive errors [34]. In the last several decades, a number of studies have evaluated the population structure of specific germplasms using limited sample sizes and sources. These studies independently provided specific information about the subpopulation differentiation of approximately 600 Minnesota maize germplasms [22], 172 Dent germplasms from Hohenheim [39], 400 maize lines from North America [23], 367 elite lines from China [19] and 527 lines representing TS and temperate backgrounds [40]. Here, we integrated maize germplasms from America, Africa, Europe and Asia, including 1857 accessions from more than 16 countries worldwide, to investigate subpopulation differentiation. The outputs of STRUCTURE V2.3.3 identified seven subpopulations: including B73, HZS, 207, Oh43, Mo17, Shen137, and TS II (Fig. 1). These results provided much more information about maize subpopulation differentiation than previous studies. In fact, the B73 (SS), Mo17 (NSS), Oh43, and 207 (IDT) subpopulations were identified using SSR markers and an Illumina MaizeSNP50 Beadchip [22]. HZS (TSPT), Shen137 (PA derived from Pioneer hybrid 78599), and TS I subpopulations were also identified in previous reports [18, 19, 41]. In addition, the findings this study was also consistent with known pedigrees. For example, LH61 shared $87.5 \%$ of its nuclear genetic material with Mo17 [42] and clustered into the Mo17 subpopulation with an ancestry membership of 0.91 (Additional file 2: Table S1). These results were consistent with those reported by Lorenz et al. [42]. Furthermore, the clustering analysis identified many more clusters, including Mo17, GB, E28, Ye8112, HZS, Shen137, PHG39, B73, B37, 207, A634, Oh43, and Reid Yellow Dent (Fig. 2). The identification of these clusters indicated that our clustering analysis increased the resolution of the categorization of accessions into subpopulations compared with the model-based method, which commonly identifies six subpopulations, Mo17, B73, HZS, Oh43, 207, and Shen137. For instance, PB80 and A632 shared $75 \%$ and $93.75 \%$ of the nuclear genetic material of B73 and B14, respectively [42], these two lines clustered into the same subpopulation as B73 and B14, respectively. This clustering was consistent with a report by Lorenz et al. [42]. Most importantly, the clustering analysis in this study identified new subpopulations that are represented by the following lines: GB, E28, Ye8112, PHG39, B37, A634, and Reid Yellow Dent. These lines correspond to the following heterotic groups: Chinese Landrace (GB) [19], Ludahonggu (E28) [41], PB (Ye8112, B37) [19] derived from modern U.S. hybrids, Commercial hybridderived lines (PHG39, A634) [10], and U.S. landrace (Reid Yellow Dent) [10], respectively. Of these groups, Chinese Landrace is mainly distributed in the northeast and southwest of China, and this variety originated from the NorthAmerican Mid-West and Mexican highlands, respectively [3]. These landraces yielded new subpopulations and have been widely used in maize-breeding programs [19]. For example, E28 is a representative line derived from crossing the landrace Ludahonggu with modified introduction lines according its pedigree [19]. Ye8112 was selected from the hybrid "8112", which originates in the U.S. [41]. Some of the lines were derived from this line, such as Ye478 and 
488, which were clustered in the heterotic group of $\mathrm{PB}$ $[19,41]$. A634 was derived from the MN13 lineage [22], is highly utilized in U.S. hybrid maize breeding. This line constituted 4.2, 7.8, and $3.0 \%$ of the total U.S. seed requirement in 1970, 1975, and 1979, respectively, and lots of lines were derived from A634 [13]. B37 is an important public line that was widely used to develop Pioneer hybrids during the 1980s [6]. The selection of a second cycle line from Pioneer hybrids resulted in new lines, which formed a subpopulation represented by B37. PHG39 is a representative inbred Maize Amargo germplasm line from which many protected corn lines have been developed. Furthermore, several important first cycle recombinant lines derived from PHG39 have been considered for commercial maize breeding [10]. These results provide maize breeders with more definitive information to effectively use historical genetic resources while maintaining the heterotic patterns necessary for hybrid breeding.

\section{Genomic differentiation and putative functions}

Genomic differentiation between subpopulations is a fundamental challenge in population genetics. Maize originated in tropical central-Mexico and rapidly spread to colder, temperate regions worldwide [32]. This diffusion caused maize to adapt to local environments by developing traits that allowed it to thrive in these environments, i.e., changes in FT and plant architecture. These changes allowed maize to reach maturity within different growing seasons. Some studies have documented the pair-wise $\mathrm{F}_{\mathrm{ST}}$ between subpopulations while considering genomic differentiation [9, 43, 44]. Schaefer and Bernardo [22] reported an average pair-wise $\mathrm{F}_{\mathrm{ST}}$ of 0.165 for one diverse panel of 284 maize inbreds; this value ranged from 0.054 between the A321 and Oh43 subpopulations to 0.325 between the Mo17 and B73 subpopulations. Romay et al. [12] found that most germplasms from classic breeding programs of the Corn Belt were closely related, with an average pair-wise $\mathrm{F}_{\mathrm{ST}}$ of 0.04. However, the differentiation regions and putative function remain poorly understood. Moreover, the $\mathrm{D}_{\mathrm{EST}}$ was also demonstrated as a measure genomic differentiation. This parameter relies on the genotypic rather than allelic number and is corrected for heterozygosity [27]; values close to zero indicate little differentiation, and values close to unity indicate nearly complete differentiation. Therefore, the $\mathrm{D}_{\mathrm{EST}}$ was used in the present study to evaluate the genomic variation between the subpopulations, and the results of this analysis revealed strong differentiation among the subpopulations. This differentiation was attributed to the continuous fixation of target genomic regions within subpopulations and strong isolation between subpopulations during maize breeding practices. The pair-wise $\mathrm{D}_{\mathrm{EST}}$ between the TS and the temperate subpopulations was 0.17 (Table 2), and 235 highly differentiated genomic regions were identified (Fig. 5). Most adaptive traits were selected and fixed during maize's long evolution and adaptation from tropical to temperate climates [31]. This fixation caused the high genomic differentiation between TS and temperate germplasms (Table 1, Figs. 2 and 3). Interestingly, 85 strongly differentiated genomic regions with a $\mathrm{D}_{\mathrm{EST}}$ exceeding 0.2 were identified between the TS and the temperate subpopulations. A genetic analysis showed that these 85 regions comprise $79 \%$ of the genetic variation of this panel (Additional file 1: Figure S4). Of these regions, 15 were significantly associated with FT-related traits based on GWAS (Fig. 4d and Additional file 1: Figure S2). In addition, two significant QTNs were closely linked to $z c n 8$ and $v g t 1$ (Fig. 4d), which are involved in maize migration and adaptation from tropical to temperate climates [31]. Beyond that, 66 highly differentiated regions were located within the interval of plant architecture or FT-related QTL (Additional file 2: Table S3). In addition, 159 highly differentiated genomic regions were also identified between SS and NSS subpopulations, with a $\mathrm{D}_{\mathrm{EST}}$ exceeding 0.16 (Fig. 5). Furthermore, 15 regions located within FT- or plant architecturerelated QTL were also identified (Additional file 2: Table S3). This finding was consistent with the marked distance between SS and NSS (Figs. 1, 2 and 3). SS and NSS are two major heterotic groups used in U.S. breeding programs that are represented by the lines B73 and Mo17, respectively. Previous studies also reported a significant distance between these two groups [23]. Furthermore, other highly differentiated genomic regions between specific subpopulation pairs were also identified, and these regions were located within a number of QTLs associated with FT- or plant architecture-related traits mapped using different bi-parental populations (Additional file 2: Table S3). In total, 303 genomic regions with a high $\mathrm{D}_{\mathrm{EST}}$ of more than 0.2 were detected, and these regions were located within 197 FT- or plant architecture-related QTLs. For example, the region containing the tag SNP PZE.108075114 was more differentiated between TS and the Temperate subpopulations $\left(D_{E S T}=0.32\right)$, and this region was located within one FT-related QTL cluster that contained the flanking markers PHTi060 and bnlg1599. These results indicate genomic regions of interest for the formation of given subpopulations and provide new insight into the dissection of the genetic basis of complex traits.

\section{Conclusions}

Here we reported that 700-1000 SNPs were necessary needed to robustly estimate the genetic differentiation of a naturally diverse panel. In addition, 13 subpopulations were identified based on genotyping and pedigree information. On this base, 85 genetic regions with higher 
differentiation between TS and temperate maize germplasm, and 197 highly differentiated regions between different subpopulation pairs were identified, which contained some FT- related QTNs/QTLs/genes supported by GWAS and linkage analysis, and some known genes of vgt1 and $z c n 8$ associated with maize adaptation from tropical to temperate belts, were also included in these regions. Therefore, we concluded that these differential regions were expected to play important roles in maize adaptation. These results would provide abundant information on the differentiation of subpopulations and new insight to help dissect the genetic basis of complex traits.

\section{Methods}

\section{Plant materials}

The present study involved an integrated diverse natural panel of 1857 accessions collected from around the world, including 400 accessions from the U.S. Department of Agriculture (USDA)'s National Plant Germplasm System [23], 280 from the North Central Regional Plant Introduction Station of the USA [45], 368 from CIMMYT [21], 48 from Africa [17], and 890 from the institute of crop sciences of the Chinese academy of agricultural sciences (ICS/CAAS). Chinese germplasm contained two sets of inbred lines: one from a previously established core [46], of 242 diverse inbred lines historically used in Chinese maize breeding and another of recently collected lines from research institutions or companies. This latter category included 648 elite inbred lines that are primarily used in current maize breeding [19]. Detailed information is listed in Additional file 2: Table S1.

\section{Phenotypic evaluation}

The FT-related traits of 1176 out of 1857 accessions were evaluated in three environments, including Beijing in 2014 (spring-sowing), Xinxiang in Henan Province in 2014 (summer-sowing), and Gongzhuling in Jilin Province in 2014 (spring-sowing). At each location, accessions were planted based on a randomized experimental design. Plants (15 plants/row) were sown in single rows that were $4 \mathrm{~m}$ long and separated by a distance of $0.6 \mathrm{~m}$. The plant density was 52,400 plants per hectare, and experiments were conducted in duplicate. FT-related traits included days to tasselling (DTT), days to silking (DTS), and days to pollen-shedding and were recorded when $50 \%$ of plants exhibited the corresponding traits. An ANOVA was performed using the PROC GLM model. A Pearson correlation analysis of FT-related traits across different environments was calculated using the PROC CORR model. The best linear unbiased predictor (BLUP) calculation was implemented using a PROC MIXED model, with genotype, location, genotype by location, and replications as random effects [47]. All above analyses were completed using the SAS software (Release 9.3; SAS Institute, Cary, NC).

\section{Genotyping datasets}

The 523 newly collected inbred lines were genotyped using a MaizeSNP50 BeadChip and 56,110 SNPs (http:// support.illumina.com/array/array_kits/maizesnp50_dna_ analysis_kit.html). When maize seedlings were one month old, the leaves of five plants were sampled in bulk to extract genomic DNA according to the modified CTAB procedure [48]. The quality of the DNA was assessed and the DNA was genotyped at the Beijing Compass Biotechnology Company according to the Infinium ${ }^{\circ} \mathrm{HD}$ assay ultra-protocol guide. In addition, the SNP genotyping datasets of the other accessions were extracted from public datasets, including those of 400 accessions submitted by van Heerwaarden et al. [23], 48 African accessions submitted by Westengen et al. [17], 368 CIMMYT accessions submitted by Li et al. [21], 280 accessions submitted by Flint-Garcia et al. [45], and 367 elite lines submitted by Wu et al. [19]. Finally, all genotypes from different panels were integrated according to the identical physical position and markers names. Alleles forms were transformed based on the pair wise base complementary. Then 43,252 SNPs were successfully obtained for the 1,857 accessions according to the following SNP screening criteria: (1) the minor allele frequency (MAF) exceeded 0.05, (2) the missing rate is less than 0.2 , and (3) the position of the marker is unambiguous on a physical map.

\section{Ascertainment bias of SNPs and PCA}

To evaluate the ascertainment bias of SNPs for evaluating the subdivision of population structure, different sample sets of SNPs were sampled across 43,252 SNPs, with window size varying from $50 \mathrm{~kb}$ to $0.2 \mathrm{Mb}$, wherein 500, 700, 800, 1000, 2000, 5000, 10,000 and 15,000 SNPs with highly genetically diverse, low missing rates, and evenly distributed across the genome were selected to do population structure analysis The subdivision of population structure for this panel was deduced with a PCA according to the method described by Patterson et al. [49] using the TASSEL software 5.0 [50]. The correlation PCs was analyzed using the SAS software (Release 9.3; SAS Institute, Cary, NC). Additionally, the average correlation coefficient of the first five PCs was used to deduce the bias extent of one given subset based on the subdivision of population structure.

\section{Model-based population structure analysis}

According the comparison of population subdividing based on different sample sets of SNP markers. A total of highly genetically diverse 5000 SNPs with low missing rates and evenly distributed across the genome were selected to estimate the population structure of the 1857 
accessions using a model-based approach [41] in STRUCTURE V2.3.3 [34]. The $\mathrm{K}$ value (the number of subpopulations) ranged from 1 to 10 , and five runs were completed for each K; the burn-in period was 5000, and 5000 replications were completed. The adhoc statistic delta $K(\Delta K)$ was used to determine the optimum number of subpopulations [51]. The outputs of STRUCTURE were integrated using CLUMPP software [52]. Subpopulation assignments were based on maximum membership probabilities for each accession [22].

\section{Neighbor-joining tree construction}

To obtain an in-depth picture of the genetic relationships between the 1857 accessions with contrasting origins, the genetic distance between accessions was calculated using 43,252 SNPs based on the modified Euclidean distance [53], which was defined as follows: $\mathrm{D}=1$ - identity by state (IBS) similarity, and the IBS was the probability that alleles derived at random from two individuals at identical loci are the same. For any two accessions, the probability of IBS was averaged over all non-missing loci. A cladogram was then constructed using the distance matrix described above based on the neighbor-joining (NJ) algorithm [54]. Clusters were identified from the resultant phylogenetic tree. All of the above calculations were conducted using the TASSEL software 5.0 [50].

\section{Basic genetic statistics}

Pair relatedness among the 1,857 accessions was estimated by definition as follows: $F_{i j}=\left(Q_{i j}-Q_{m} /\left(1-Q_{m}\right)\right.$, where $\mathrm{Q}_{\mathrm{ij}}$ is the probability of IBS for random loci from $i$ and $j$, and $Q_{m}$ is the average probability of IBS for loci from random individuals. Pair-kinship coefficients of 0 , 0 to 0.1 and 0.1 to 0.5 indicated weak, intermediate, and strong similarity between accessions, respectively [35]. The GD, heterozygosity, and PIC were calculated in PowerMarker V3.25 [55], with heterozygosity being defined as the proportion of heterozygous loci detected in a single accession, and GD being defined as the probability that two alleles randomly chosen from the test sample were different [18]. The PIC was estimated as follows: $\quad \mathrm{PIC}_{l}=1-\sum_{u=1}^{k} \tilde{\mathrm{p}}_{\mathrm{lu}}^{2}-\sum_{u=1}^{k-1} \sum_{v=u+1}^{k} 2 \tilde{\mathrm{p}}_{\mathrm{lu}}^{2} \tilde{\mathrm{p}}_{\mathrm{lv}}^{2}$, where $\mathrm{p}_{\mathrm{lu}}$ and $\mathrm{p}_{\mathrm{lv}}$ are the frequencies of the $u$ th and $v$ th alleles for marker $l$, respectively. The LD level between SNPs was evaluated using the squared Pearson correlation coefficient $\left(\mathrm{r}^{2}\right)$ between vectors of SNP alleles according to a previous study [56]. This evaluation was completed in the TASSEL software 5.0 [50]. The $\mathrm{D}_{\mathrm{EST}}$ values were calculated following the algorithm described by Yang [57] using the $\mathrm{R}$ 'hierfstat' package (http:// cran.r-project.org/web/packages/hierfstat). The $\mathrm{D}_{\mathrm{EST}}$ was defined as $\mathrm{D}_{\text {EST }}=\left[\left(\mathrm{H}_{\text {Test }}-\mathrm{H}_{\text {Sest }}\right) /\left(1-\mathrm{H}_{\text {Sest }}\right)\right] /[\mathrm{n} /(\mathrm{n}-1)]$, where $\mathrm{H}_{\text {Sest }}$ is the observed gene diversity within subpopulation,
$\mathrm{H}_{\text {Test }}$ is the overall gene diversity, and $\mathrm{n}$ is the number of subpopulations [27]. The size of genomic regions was defined as window size with double the value of the LD level of this panel, and the average $\mathrm{D}_{\mathrm{EST}}$ of all SNPs in LD was defined as the cutoff value to interpret the relevant differentiation level between subpopulations. Genomic regions with an average $D_{\text {EST }}$ exceeding the cutoff value were treated as cases of strong differentiation between subpopulations.

\section{Genome wide association study}

The BLUP of FT-related traits, including the DTT, DTP and DTS for each accession across three environments, and 43,252 SNPs were selected to perform a phenotypegenotype GWAS, which was implemented in the GAPIT $\mathrm{R}$ package [58], using mix linear model (MLM) in which the population structure and pair-kinship were treated as covariates [38]. The significant cutoff value was defined as 0.05 divided by the number of markers. QTNs were selected for further analysis when the $P$-values of SNPs were less than the cutoff value.

\section{Availability of supporting data}

All data sets supporting the results of this article are included within the article.

\section{Additional files}

Additional file 1: Figure S1. Correlation analysis of the first five principal components (PCs) between specific subset with different marker size and the entire set with all markers. Figure S2. Pair-comparison of $D_{\text {EST }}$ on different chromosomes between the subpopulations. Figure S3. Summary statistic of flowering time related traits. Histograms show the distribution of flowering time related traits. Numbers in the up-right triangle are the Pearson coefficient between flowering time related traits and between environments for the same trait. The low-left triangle is the scatter plots between flowering time related traits and between environments for the same trait. Figure S4. Comparison of the genetic variation evaluated by using two datasets including 43,252 and 85 SNPs, respectively. Each scatter presents one accession. Black line is the fitted of all scatters. (ZIP $850 \mathrm{~kb}$ )

Additional file 2: Table S1. Summary information of sources and co-ancestral membership of 1857 accessions Table S2. GWAS results of flowering time related traits and relevant linked QTL or genes. Table S3. Genomic regions with DEST of more than 0.2 and relevant linked QTL. (XLSX 299 kb)

\section{Abbreviation}

SNP: Single-nucleotide polymorphisms; HZS: Huangzaosi; TS: Tropical/ subtropical; FT-: Flowering time related traits; GWAS: Genome-wide association study; QTL: Quantitative trait loci; SS: lowa Stiff Stalk Synthetic; NSS: Non-Stiff Stalk; IDT: lodent; GBS: Genotyping by sequencing; PC: Principal components; $\triangle K$ : delta K; MICN: Modified Introduction in China; LD: Linkage disequilibrium; GDs: Gene diversities; PICs: Polymorphism information contents; DTT: Days to tasseling; DTS: Days to silking; and DTP: Pollen-shedding; MLM: Mix linear model; IBS: Identity by state; BLUP: Best linear unbiased predictor; GLM: General line model.

\section{Competing interests}

The authors declared that they have no competing interests. 


\section{Authors' contribution}

$X W, X L$ and $C L$ carried out phenotyping and genotyping of diverse inbred lines. XW, implemented all data collection, analysis and wrote this manuscript. $X W, Y L, T W, Y S, Y S, Z Z$ and $Y L$ conceived of this study and participated in its design and coordination and helped to draft the manuscript. All authors had read and approved the final manuscript.

\section{Acknowledgments}

Some genotyping datasets used in this paper were reported by van

Heerwaarden et al. [23], Westengen et al. [17], Li et al. [21], and Flint-Garcia et al. [45]. We thank them and their colleagues for sharing their genotyping data. This research was supported by the Ministry of Sciences and Technology of China (2014CB138200 and 2011DFA30450), the National Natural Science Foundation (91335206), the China Postdoctoral Sciences Foundation (2014M550901), and the CAAS Innovation Program.

\section{Author details}

${ }^{1}$ Institute of Crop Science, Chinese Academy of Agricultural Science, Beijing, China. ${ }^{2}$ Nanchong Academy of Agricultural Sciences, Nanchong, Sichuan, China.

\section{Received: 20 August 2015 Accepted: 15 October 2015}

\section{Published online: 24 October 2015}

\section{References}

1. Wang TY, Ma XL, Li Y, Bai DP, Liu C, Liu ZZ, et al. Changes in yield and yield components of single-cross maize hybrids released in china between 1964 and 2001. Crop Science. 2011:51(2):512-25.

2. Matsuoka Y, Vigouroux Y, Goodman MM, Sanchez GJ, Buckler E, Doebley J. A single domestication for maize shown by multilocus microsatellite genotyping. Proc Natl Acad Sci U S A. 2002;99(9):6080-4.

3. Mir C, Zerjal T, Combes V, Dumas F, Madur D, Bedoya C, et al. Out of America: tracing the genetic footprints of the global diffusion of maize. Theor Appl Genet. 2013;126(11):2671-82.

4. $\quad$ Yang $Z$, Zhang E, Li J, Jiang $Y$, Wang $Y$, Hu Y, et al. Analyses of sequence polymorphism and haplotype diversity of LEAFY genes revealed postdomestication selection in the Chinese elite maize inbred lines. Mol Biol Rep. 2014;41(2):1117-25.

5. Dubreuil P, Warburton M, Chastanet M, Hoisington D, Charcosset A. More on the introduction of temperate maize into Europe: Large-scale bulk SSR genotyping and new historical elements. Maydica. 2006;51(2):281-91.

6. Smith S. Pedigree background changes in US hybrid maize between 1980 and 2004. Crop Science. 2007:47(5):1914-26.

7. Bracco M, Lia W, Hernandez JC, Poggio L, Gottlieb AM. Genetic diversity of maize landraces from lowland and highland agro-ecosystems of Southern South America: implications for the conservation of native resources. Ann Appl Biol. 2012;160(3):308-21.

8. Hallauer AR. Evolution of plant breeding. Crop Breed Appl Biot. 2011;11(3):197-206.

9. Jiao Y, Zhao H, Ren L, Song W, Zeng B, Guo J, et al. Genome-wide genetic changes during modern breeding of maize. Nat Genet. 2012;44(7):812-5.

10. Mikel MA, Dudley JW. Evolution of north American dent corn from public to proprietary germplasm. Crop Science. 2006;46(3):1193-205.

11. Schnable JC. Genome evolution in maize: from genomes back to genes. Annu Rev Plant Biol. 2015;66:329-43.

12. Romay MC, Millard MJ, Glaubitz JC, Peiffer JA, Swarts KL, Casstevens TM, et al. Comprehensive genotyping of the USA national maize inbred seed bank. Genome Biol. 2013;14(6):R55.

13. Troyer AF, Hendrickson LG. Background and importance of 'Minnesota 13' corn. Crop Science. 2007;47(3):905-14

14. Peng B, Li Y, Wang Y, Liu C, Liu Z, Tan W, et al. QTL analysis for yield components and kernel-related traits in maize across multi-environments. Theor Appl Genet. 2011;122(7):1305-20.

15. Upadyayula N, da Silva HS, Bohn MO, Rocheford TR. Genetic and QTL analysis of maize tassel and ear inflorescence architecture. Theor Appl Genet. 2006;112(4):592-606.

16. Vidal-Martinez VA, Clegg MD, Johnson BE. Genetic studies on maize pollen and grain yield and their yield components. Maydica. 2001:46(1):35-40.

17. Westengen OT, Berg PR, Kent MP, Brysting AK. Spatial structure and climatic adaptation in African maize revealed by surveying SNP diversity in relation to global breeding and landrace panels. Plos One. 2012;7(10), e47832.
18. Lu YL, Yan JB, Guimaraes CT, Taba S, Hao ZF, Gao SB, et al. Molecular characterization of global maize breeding germplasm based on genome-wide single nucleotide polymorphisms. Theor Appl Genet. 2009;120(1):93-115.

19. Wu X, Li Y, Shi Y, Song Y, Wang T, Huang Y, et al. Fine genetic characterization of elite maize germplasm using high-throughput SNP genotyping. Theor Appl Genet. 2014;127(3):621-31.

20. Yan J, Shah T, Warburton ML, Buckler ES, McMullen MD, Crouch J. Genetic characterization and linkage disequilibrium estimation of a global maize collection using SNP markers. Plos One. 2009;4(12), e8451.

21. Li H, Peng Z, Yang X, Wang W, Fu J, Wang J, et al. Genome-wide association study dissects the genetic architecture of oil biosynthesis in maize kernels. Nat Genet. 2013;45(1):43-50.

22. Schaefer CM, Bernardo R. Population Structure and Single Nucleotide Polymorphism Diversity of Historical Minnesota Maize Inbreds. Crop Science. 2013:53(4):1529

23. van Heerwaarden J, Hufford MB, Ross-Ibarra J. Historical genomics of North American maize. Proc Natl Acad Sci. 2012;109(31):12420-5.

24. Hoisington D, Khairallah M, Reeves T, Ribaut JV, Skovmand B, Taba S, et al. Plant genetic resources: What can they contribute toward increased crop productivity? Proc Natl Acad Sci U S A. 1999;96(11):5937-43.

25. Lee M. Genome projects and gene pools: New germplasm for plant breeding? Proc Natl Acad Sci U S A. 1998;95(5):2001-4.

26. Weller SG, Sakai AK. Using phylogenetic approaches for the analysis of plant breeding system evolution. Annu Rev Ecol Syst. 1999;30:167-99.

27. Jost LOU. GSTand its relatives do not measure differentiation. Mol Ecol. 2008;17(18):4015-26.

28. Haag T, Santos AS, Sana DA, Morato RG, Cullen L, Crawshaw PG, et al. The effect of habitat fragmentation on the genetic structure of a top predator: loss of diversity and high differentiation among remnant populations of Atlantic Forest jaguars (Panthera onca). Mol Ecol. 2010;19(22):4906-21.

29. Liu H, Wang X, Warburton ML, Wen W, Jin M, Deng M, et al. Genomic, transcriptomic, and phenomic variation reveals the complex adaptation of modern maize breeding. Mol Plant. 2015;8(6):871-84.

30. Hufford MB, Xu X, van Heerwaarden J, Pyhajarvi T, Chia JM, Cartwright RA et al. Comparative population genomics of maize domestication and improvement. Nat Genet. 2012:44(7):808-U118.

31. Bouchet S, Servin B, Bertin P, Madur D, Combes V, Dumas F, et al. Adaptation of maize to temperate climates: Mid-density genome-wide association genetics and diversity patterns reveal key genomic regions, with a major contribution of the Vgt2 (ZCN8) locus. Plos One. 2013;8(8), e71377.

32. Ducrocq S, Madur D, Veyrieras JB, Camus-Kulandaivelu L, Kloiber-Maitz M, Presterl T, et al. Key impact of Vgt1 on flowering time adaptation in maize: Evidence from association mapping and ecogeographical information. Genetics. 2008;178(4):2433-7.

33. Morin PA, Martien KK, Taylor BL. Assessing statistical power of SNPs for population structure and conservation studies. Mol Ecol Resour. 2009;9(1):66-73.

34. Pritchard JK, Stephens M, Donnelly P. Inference of population structure using multilocus genotype data. Genetics. 2000;155(2):945-59.

35. Yang XH, Xu YB, Shah T, Li HH, Han ZH, Li JS, et al. Comparison of SSRs and SNPs in assessment of genetic relatedness in maize. Genetica. 2011;139(8):1045-54.

36. Hamblin MT, Warburton ML, Buckler ES. Empirical comparison of Simple Sequence Repeats and single nucleotide polymorphisms in assessment of maize diversity and relatedness. Plos One. 2007;2(12), e1367.

37. Yu JM, Zhang ZW, Zhu CS, Tabanao DA, Pressoir G, Tuinstra MR, et al. Simulation appraisal of the adequacy of number of background markers for relationship estimation in association mapping. Plant genome. 2009;2(1):63-77.

38. Yu JM, Pressoir G, Briggs WH, Bi IV, Yamasaki M, Doebley JF, et al. A unified mixed-model method for association mapping that accounts for multiple levels of relatedness. Nat Genet. 2006;38(2):203-8.

39. Technow F, Schrag TA, Schipprack W, Melchinger AE. Identification of key ancestors of modern germplasm in a breeding program of maize. Theor Appl Genet. 2014;127(12):2545-53.

40. Yang XH, Gao SB, Xu ST, Zhang ZX, Prasanna BM, Li L, et al. Characterization of a global germplasm collection and its potential utilization for analysis of complex quantitative traits in maize. Mol Breeding. 2011;28(4):511-26.

41. Wang R, Yu Y, Zhao J, Shi $Y$, Song $Y$, Wang T, et al. Population structure and linkage disequilibrium of a mini core set of maize inbred lines in China. Theor Appl Genet. 2008;117(7):1141-53. 
42. Lorenz A, Hoegemeyer T. The phylogenetic relationships of US maize germplasm. Nat Genet. 2013;45(8):844-5.

43. Loaisiga CH, Rocha O, Brantestam AK, Salomon B, Merker A. Genetic diversity and gene flow in six accessions of Meso-America teosintes. Genet Resour Crop Ev. 2012;59(1):95-111.

44. Wen W, Franco J, Chavez-Tovar VH, Yan J, Taba S. Genetic characterization of a core set of a tropical maize race Tuxpeno for further use in maize improvement. Plos One. 2012;7(3), e32626

45. Flint-Garcia SA, Thuillet AC, Yu JM, Pressoir G, Romero SM, Mitchell SE, et al. Maize association population: a high-resolution platform for quantitative trait locus dissection. Plant J. 2005;44(6):1054-64

46. Li Y, Shi YS, Cao YS, Wang TY. Establishment of a core collection for maize germplasm preserved in Chinese National Genebank using geographic distribution and characterization data. Genet Resour Crop Ev. 2004;51(8):845-52.

47. Brown PJ, Upadyayula N, Mahone GS, Tian F, Bradbury PJ, Myles S, et al. Distinct genetic architectures for male and female inflorescence traits of maize. PLoS genetics. 2011;7(11), e1002383.

48. Saghai-Maroof MA, Soliman KM, Jorgensen RA, Allard RW. Ribosomal DNA spacer-length polymorphisms in barley: mendelian inheritance, chromosomal location, and population dynamics. Proc Natl Acad Sci U S A. 1984;81(24):8014-8.

49. Patterson N, Price AL, Reich D. Population structure and eigenanalysis. PLoS genetics. 2006;2(12):2074-93.

50. Bradbury PJ, Zhang Z, Kroon DE, Casstevens TM, Ramdoss Y, Buckler ES. TASSEL: software for association mapping of complex traits in diverse samples. Bioinformatics. 2007;23(19):2633-5.

51. Evanno G, Regnaut $S$, Goudet J. Detecting the number of clusters of individuals using the software STRUCTURE: a simulation study. Mol Ecol. 2005;14(8):2611-20.

52. Jakobsson M, Rosenberg NA. CLUMPP: a cluster matching and permutation program for dealing with label switching and multimodality in analysis of population structure. Bioinformatics. 2007;23(14):1801-6.

53. Legesse BW, Myburg AA, Pixley KV, Botha AM. Genetic diversity of African maize inbred lines revealed by SSR markers. Hereditas. 2007:144(1):10-7.

54. Zhang W, Sun ZR. Random local neighbor joining: A new method for reconstructing phylogenetic trees. Mol Phylogenet Evol. 2008:47(1):117-28

55. Liu KJ, Muse SV. PowerMarker: an integrated analysis environment for genetic marker analysis. Bioinformatics. 2005;21 (9):2128-9.

56. Hill WG, Weir BS. Maximum-likelihood estimation of gene location by linkage disequilibrium. Am J Hum Genet. 1994;54(4):705-14.

57. Yang RC. Estimating hierarchical F-statistics. Evolution. 1998;52(4):950-6.

58. Lipka AE, Tian F, Wang QS, Peiffer J, Li M, Bradbury PJ, et al. GAPIT: genome association and prediction integrated tool. Bioinformatics. 2012;28(18):2397-9.

\section{Submit your next manuscript to BioMed Central and take full advantage of:}

- Convenient online submission

- Thorough peer review

- No space constraints or color figure charges

- Immediate publication on acceptance

- Inclusion in PubMed, CAS, Scopus and Google Scholar

- Research which is freely available for redistribution 